\title{
Citrullinated Calreticulin Potentiates Rheumatoid Arthritis Shared Epitope Signaling
}

\author{
Song Ling, Erika N. Cline, Timothy S. Haug, David A. Fox, and Joseph Holoshitz
}

Objective. Citrullinated proteins are immunogenic in rheumatoid arthritis (RA), particularly in patients who carry shared epitope (SE)-coding HLADRB1 alleles. The mechanism underlying this association is unknown. We have previously identified the $\mathrm{SE}$ as a ligand that interacts with cell surface calreticulin (CRT) and activates immune dysregulation. This study was undertaken to determine the effect of CRT citrullination on SE signaling.

Methods. CRT-SE binding affinity was measured by surface plasmon resonance. The role of individual CRT arginine residues was determined by site-directed mutagenesis, and nitric oxide levels were measured using a fluorochrome-based assay. CRT citrullination in synovial tissue samples and cell cultures was determined by 2-dimensional gel electrophoresis, immunoblotting, and mass spectrometry techniques.

Results. Synovial tissue and fibroblast-like synoviocytes from RA patients were found to express a higher abundance of citrullinated CRT than samples from osteoarthritis patients. Citrullinated CRT showed more robust interaction with the SE ligand, and transduced SE signaling at a 10,000-fold higher potency, compared to noncitrullinated CRT. Site-directed mutation analysis identified $\mathrm{Arg}^{205}$, which is spatially adjacent to the SE binding site in the CRT P-domain, as a

Dr. Fox's work was supported by the NIH (grant AR-38477). Dr. Holoshitz' work was supported by the NIH (grants GM-088560, AR-059085, AR-056786, and AR-55170) and by an Innovative Basic Science Award from the Rheumatology Research Foundation of the American College of Rheumatology.

Song Ling, PhD, Erika N. Cline, BA, Timothy S. Haug, BS, David A. Fox, MD, Joseph Holoshitz, MD: University of Michigan School of Medicine, Ann Arbor.

Drs. Ling and Holoshitz are named inventors on six patents and two pending patent applications for devices/substances/procedures related to rheumatoid arthritis. The patents are owned by the University of Michigan, and Drs. Ling and Holoshitz receive no licensing fees. Address correspondence to Joseph Holoshitz, MD, University of Michigan, 5520D MSRB1, Box 0680, 1150 West Medical Center Drive, Ann Arbor, MI 48109-5680. E-mail: jholo@umich.edu.

Submitted for publication June 26, 2012; accepted in revised form November 27, 2012. dominant inhibitor of SE-CRT interaction and signaling, while a more remote arginine residue, $\mathrm{Arg}^{261}$, was found to enhance these SE functions.

Conclusion. Our findings indicate that citrullinated CRT is overabundant in the RA synovium and potentiates SE-activated signaling in vitro. These findings could introduce a new mechanistic model of geneenvironment interaction in RA.

The shared epitope (SE), a 5-amino acid sequence motif in positions $70-74$ of the HLA-DR $\beta$ chain, is the most significant genetic risk factor for rheumatoid arthritis (RA) (1,2). The disease is more erosive in individuals carrying SE-coding HLA-DRB1 alleles than in SE-negative individuals (3). The mechanism underlying the effect of the SE in RA is unknown. We have recently identified the $\mathrm{SE}$ as a ligand that activates innate signaling and Th17 polarization (4-7). Whether expressed in its native conformation on the cell surface, as a cell-free HLA-DR tetrameric molecule, engineered into large recombinant proteins, or as a short synthetic peptide, the SE activates nitric oxide (NO) production in a strictly allele-specific manner. SEactivated signaling is transduced via cell surface calreticulin (CRT) (6), an established innate immunity receptor. Using a point mutagenesis technique, we have recently mapped the $\mathrm{SE}$ binding site to amino acid residues $\mathrm{Glu}^{217}$, $\mathrm{Asp}^{220}$, and $\mathrm{Glu}^{223}$ in the CRT P-domain (8).

Although genetic factors strongly influence disease risk, the concordance rate of RA in identical twins is only $15 \%$ (9). It has therefore been proposed that while susceptibility is determined genetically, disease onset may depend on environmentally triggered stochastic events, such as posttranslational protein modifications (for review, see ref. 10). One of the better-known protein modifications in RA involves deimination of arginine residues to citrulline by peptidyl arginine deiminase (PAD). Citrullinated proteins are overabundant in 
RA joints, as well as many other inflammatory tissues, such as the brain in patients with multiple sclerosis, muscle in patients with myositis, or the gut in patients with Crohn's disease $(11,12)$.

Several citrullinated proteins have been identified in RA (13-15), and some of them have been found to be targets of anti-citrullinated protein antibodies (ACPAs), which are useful biomarkers for RA (for review, see ref. 16). Although the pathogenic role of ACPAs in human RA remains an open question, it is well established that these antibodies are more commonly found in SE-positive RA patients (16). Furthermore, an SE gene-dose effect on RA risk has been documented in ACPA-positive RA patients (17).

Thus, RA development involves an intricate relationship between genetic (notably SE) and acquired, nongenetic (protein citrullination) factors. Several studies have identified synergism between SE and smoking in RA (18-20), and an association between smoking and pulmonary PAD-2 expression, supporting the hypothesis that smoking-induced PAD activation may trigger the onset of an SE-driven disease (21).

Given the known association of the SE with RA, its recently discovered signaling effect via CRT, the association of protein citrullination with the disease, and the interaction between the SE and a citrullinationdriven RA biomarker, we have sought to determine whether CRT citrullination affects SE signaling. Here we demonstrate that citrullinated CRT (Cit-CRT) has higher affinity for the SE ligand with resultant potentiation of SE-activated signaling. Using point mutagenesis, we have identified 3 arginine residues in CRT that modulate SE binding to CRT and, consequently, the potency of its signaling. Finally, we demonstrate that Cit-CRT is overabundant in RA synovial tissue. These results provide new insights into the functional effect of the SE and identify a candidate molecular basis for a mechanistic model of gene-environment interaction in RA.

\section{MATERIALS AND METHODS}

Cells and reagents. The mouse embryonic fibroblast (MEF) line K42, isolated from $\mathrm{crt}^{-1-}$ mice, has been described previously (22). Diaminofluorescein diacetate (DAF-2 DA) was purchased from EMD Calbiochem. SE ligands were expressed as either 15-mer synthetic peptides $(65-79 * 0401$ or $65-79 * 0402)$ or in the form of HLA-DR4 tetramers as previously described (4-8). All other chemicals were from Sigma. Fibroblast-like synoviocytes (FLS) were prepared as previously described (22) from synovial tissue obtained from patients with RA or osteoarthritis (OA) who were undergoing joint replacement surgery at the University of Michigan Health system.
FLS were used between passages 4 and 8 . Patients classified as having RA satisfied the 1987 American College of Rheumatology criteria (23). The presence of OA was ascertained by clinical diagnosis and characteristic findings on radiographs. Procedures involving human subjects were conducted under an institutional review board-approved protocol.

Generation of CRT point mutants. Site-directed mutagenesis was performed following the QuikChange protocol (Stratagene). Sequences were verified by the University of Michigan DNA Sequencing Core. Mouse wild-type CRT and its site-directed mutants were cloned into $\mathrm{pBAD} / \mathrm{glll} \mathrm{A-His}$ plasmid as previously described (24). Wild-type and mutant CRT plasmids were transformed into GC10 cells for protein expression. The $6 \times$ His-tagged protein expression was induced by incubation with $0.002 \%$ L-arabinose for 4 hours, and the protein was purified using a nickel-nitrilotriacetic acid resin following the recommendations of the manufacturer (Qiagen).

In vitro citrullination of CRT. Purified mouse CRT was incubated with 2 units of PAD from rabbit skeleton muscle in the reaction buffer $\left(0.1 M\right.$ Tris $\mathrm{HCl}$, pH7.6, $10 \mathrm{~m} M \mathrm{CaCl}_{2}, 5$ $\mathrm{m} M$ dithioerythritol, and $1 \times$ proteinase inhibitor cocktail) for 16 hours at $37^{\circ} \mathrm{C}(25)$. At the end of incubation, the reaction mixture was loaded onto $10 \%$ sodium dodecyl sulfate (SDS)polyacrylamide gel electrophoresis gel. The CRT band was in-gel digested by Glu-c. Then citrullination sites on CRT were confirmed using an LTQ Orbitrap XL (as described below) at the Proteomic Resource Facility in the Department of Pathology, University of Michigan.

Surface plasmon resonance. A BIAcore 2000 instrument (GE Healthcare) was used as previously described $(6,8)$. All assays were performed at $25^{\circ} \mathrm{C}$ in a binding buffer containing $10 \mathrm{~m} M$ HEPES (pH 7.4), $50 \mathrm{~m} M \mathrm{KCl}, 0.5 \mathrm{mM} \mathrm{CaCl}{ }_{2}, 100$ $\mu M \mathrm{ZnCl}_{2}$, and $0.005 \%$ Surfactant P20. The analyte was injected at a flow rate of $10 \mu \mathrm{l} /$ minute.

Chemical modification of citrulline residues. Cell lysates were chemically modified in reaction mixture with 8.3 $\mathrm{m} M$ antipyrine, $50 \%$ trifluoroacetic acid, and $8.3 \mathrm{~m} M$ 2,3butanedione, as previously described (26). The mixture was incubated for 2 hours at $37^{\circ} \mathrm{C}$ in the dark. At the end of modification, the reaction mixture leftover was removed by desalting column. The chemically modified cell lysates were processed by immunoprecipitation.

Mass spectrometry (MS). MS analysis of in vitro citrullinated CRT was performed by the liquid chromatography electrospray ionization MS/MS method at the Proteomic Resource Facility in the Department of Pathology, University of Michigan. Peptide samples were suspended in $1 \%$ acetic acid and $2 \%$ acetonitrile, and loaded onto a reverse-phase separation column $(0.075 \times 100 \mathrm{~mm})$ packed in house with MAGIC C18 AQ $5 \mu \mathrm{m}$ (Michrom Bioresources). The peptides were separated on a $1 \%$ acetic acid/acetonitrile gradient system (5-50\% acetonitrile for 75 minutes, followed by a 10 minute $95 \%$ acetonitrile wash) at a flow rate of $\sim 300 \mathrm{nl} /$ minute. Peptides were directly sprayed onto the MS using a nanospray source. An LTQ Orbitrap XL (Thermo Fisher Scientific) was run in automatic mode collecting a highresolution MS scan (full-width half-maximum 30,000) followed by data-dependent acquisition of MS/MS scans on the 9 most intense ions (relative collision energy $\sim 35 \%$ ). Dynamic exclusion was set to collect $2 \mathrm{MS} / \mathrm{MS}$ scans on each ion and exclude 
it for an additional 2 minutes. Charge state screening was enabled to exclude +1 and undetermined charge states (27). Raw data were searched against the UniProt mouse protein database (released May 2011) appended with reverse sequences using the X!Tandem/TPP software suite. Carbamidomethylation of cysteine (57.0214 daltons), oxidation of methionine (15.9949 daltons), and citrullination of arginine ( +0.9840 daltons) were considered as potential modifications. Precursor mass tolerance of 50 parts per million, and fragment mass tolerance of 0.5 daltons were allowed. Peptides containing citrullinated arginine with a PeptideProphet probability of $>0.9$ were considered positive and manually verified.

To identify CRT citrullination sites in synovial tissue and FLS, mass spectrometric analysis of chemically modified citrulline residues was performed at the University of Michigan Proteome Mapping Core. The matrix-assisted laser desorption ionization-time-of-flight/time-of-flight mass spectra of chymotrypsin-digested peptide were obtained using an Applied Biosystems 4800 Proteomics Analyzer, as previously described (8).

Immunoprecipitation analysis. Tissue or cell lysates were precleared by incubation with protein $\mathrm{G}$-agarose beads (Sigma) for 15 minutes at $4^{\circ} \mathrm{C}$. The precleared part was then incubated overnight with beads and a 1:25 dilution of mouse anti-CRT antibody (Becton Dickinson). Beads were then washed once with radioimmunoprecipitation assay buffer and twice with phosphate buffered saline, and the immune complexes were released from the beads by boiling in sample buffer for 5 minutes. Following SDS-polyacrylamide gel electrophoresis, immunoprecipitated products were analyzed by Western blotting using anticitrulline antibody (Abcam). The CRT level on the same blot was confirmed by rabbit anti-CRT antibody (Pierce).

Two-dimensional gel electrophoresis (2-DE). RA and OA FLS were analyzed by Western blotting using 2-DE membranes. Proteins were focused at $20^{\circ} \mathrm{C}$, with $11 \mathrm{~cm}, \mathrm{pH}$ 3-5.6, immobilized $\mathrm{pH}$ gradient ReadyStrip IPG strips (Bio$\mathrm{Rad})$, which had been incubated in $200 \mu \mathrm{l}$ protein extract mixed with rehydration buffer ( $8 M$ urea, $2 \%$ CHAPS, $1 \%$ dithiothreitol [DTT], trace of bromophenol blue, $0.2 \%$ Biolyte carrier ampholytes [pH 3-5.6]) (Bio-Rad) for 16 hours. A Protean IEF cell (Bio-Rad) was used with fast-voltage ramping at a maximum voltage of $6,000 \mathrm{~V}$ for 20 hours. After the first-dimension run, the strips were equilibrated by incubation in $6 M$ urea, $0.375 M$ Tris $\mathrm{HCl}(\mathrm{pH} 8.8), 2 \%$ SDS, $20 \%$ glycerol, $2.5 \%$ weight/volume DTT at $10 \mathrm{ml}$ per strip for 20 minutes at room temperature, followed by an incubation for 30 minutes in the same buffer but in which DTT was replaced by $2.5 \% \mathrm{w} / \mathrm{v}$ iodoacetamide. Strips were then placed on the top of $4-12 \%$ Criterion XT precast gels $(11 \mathrm{~cm} \times 8 \mathrm{~cm} \times 1 \mathrm{~mm})($ Bio-Rad) and migrated constantly at $200 \mathrm{~V}$ until the bromophenol blue dye front had reached the bottom of the gel. A BenchMark prestained protein ladder (Invitrogen) was used as the molecular weight standard in the second-dimension step. Finally, gels were either stained with Sypro Ruby protein gel stain (Bio-Rad) or were electroblotted onto nitrocellulose membranes. Sypro Ruby-stained 2-D gels were scanned using a densitometer, and Western blotting analyses were performed.

Signal transduction assays. To measure NO production, $\mathrm{crt}^{-/-}$MEF were seeded in a 96-well plate at 30,000 cells per well. Cultures were loaded with recombinant WT or mutant CRT overnight. Cells were then labeled by $20 \mu M$
DAF-2 DA for 1 hour and stimulated with SE ligands. NO production rates were determined as previously described (4-8).

Statistical analysis. Data are expressed as the mean \pm SEM from triplicate samples. Statistical analyses were performed using Student's 2-tailed $t$-test.

\section{RESULTS}

CRT citrullination enhances SE-CRT interaction and potentiates SE-activated signaling. RA epidemiology and translational research findings strongly suggest that while RA susceptibility is determined genetically, disease onset may depend on environmentally triggered stochastic events, such as posttranslational protein modifications. Given the growing evidence for aberrant protein citrullination in RA, we first examined whether citrullination of CRT could affect the functional properties of the SE.

Recombinant CRT was citrullinated in vitro as previously reported (25). To determine the effect of global CRT citrullination on its interaction with the SE, Cit-CRT or native CRT proteins were immobilized on biosensor chips and their binding affinities toward SE peptides, or SE-expressing HLA-DR tetramers, were determined. As can be seen in Figure 1, the SE ligand 65-79*0401 (a 15-mer peptide corresponding to the 65-79 region, coded by an SE-positive allele, DRB $1 * 04$ : 01 ) interacted more potently with Cit-CRT than with native CRT. A control peptide, 65-79*0402 (corresponding to the 65-79 region, coded by an SE-negative allele, DRB1*04:02), did not bind to either native CRT or Cit-CRT. The SE-positive HLA tetramer T-0401 (4 units of heterodimeric molecules, each made of a $\mathrm{DR} \beta$ chain coded by allele HLA-DRB1*04:01 and a DR $\alpha$ chain coded by HLA-DRA1) showed markedly higher interaction with Cit-CRT than with native CRT. A control, SE-negative tetramer T-1501, did not bind to native CRT and did not show preferential binding to Cit-CRT (Figure 1B). Kinetics analysis (Table 1) revealed that Cit-CRT had 100 -fold higher affinity $\left(K_{\mathrm{D}}\right)$ for the SE ligand compared with native CRT. Importantly, the affinity of C1q, another CRT ligand, was unaffected by citrullination. Thus, we conclude that global citrullination of CRT potentiates its interaction with the SE ligand.

We next performed experiments to determine whether increased affinity in cell-free binding studies has functional correlates in signal transduction assays. We $(6,8)$ and others $(28,29)$ have previously demonstrated that when soluble CRT is added to CRTnegative cells it attaches to the cell surface and restores CRT receptor-mediated signaling. Accordingly, to de- 


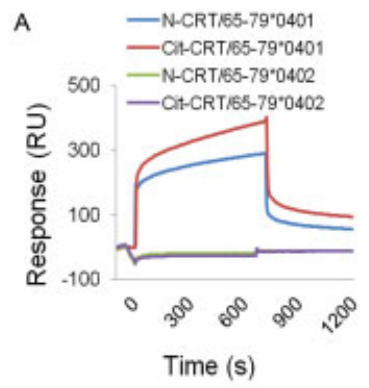

C

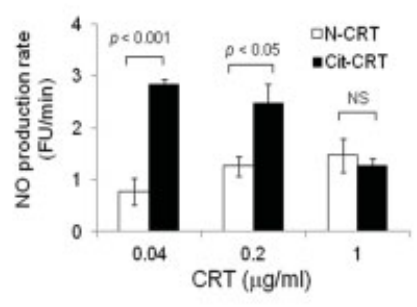

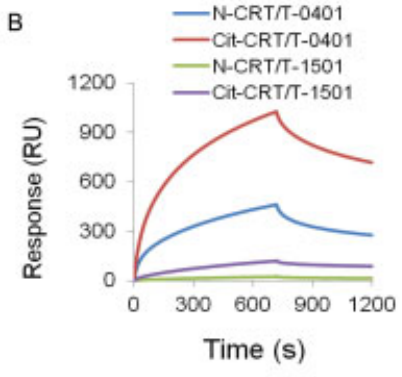

D

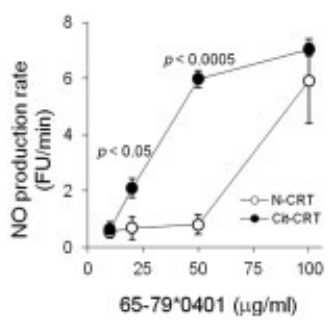

Figure 1. Calreticulin (CRT) citrullination enhances the interaction between CRT and the shared epitope (SE) and potentiates SEactivated signaling. A, Surface plasmon resonance (SPR) data showing binding interactions between native CRT (N-CRT) or citrullinated CRT (Cit-CRT) and the SE-positive peptide ligand $65-79 * 0401$ or the control SE-negative peptide $65-79 * 0402$. The native CRT and CitCRT proteins were immobilized on a biosensor chip at 3,000 response units (RU), 65-79*0401 and $65-79 * 0402$ were each applied in the analyte at $330 \mu M$, and binding interactions were determined. B, SPR data showing binding interactions between native CRT or Cit-CRT and SE-positive (T-0401) or SE-negative (T-1501) tetramers each applied in the analyte at a $250 \mathrm{n} M$ concentration. $\mathbf{C}$, Nitric oxide (NO) production by CRT-deficient cells incubated with various concentrations of native CRT or Cit-CRT and $100 \mu \mathrm{g} / \mathrm{ml}$ of the SE-positive peptide $65-79 * 0401$. D, NO production by $\mathrm{crt}^{-1-}$ mouse cells incubated with $1 \mu \mathrm{g} / \mathrm{ml}$ of native CRT or Cit-CRT and various concentrations of the SE-positive peptide $65-79^{*} 0401$. Data are representative of at least 2 independent identical experiments. Values in $\mathbf{C}$ and $\mathbf{D}$ are the mean \pm SEM. FU $=$ fluorescence units; NS $=$ not significant.

termine the effect of CRT citrullination on signal transduction, soluble native CRT or Cit-CRT proteins were added to CRT-deficient cells, followed by stimulation with the SE ligand. As can be seen, Cit-CRT mediated signal transduction much more effectively. It transduced SE signaling at significantly lower receptor concentrations (Figure 1C) and at a much lower ligand concentration threshold (Figure 1D) than the native CRT. The $50 \%$ maximum response concentration of SE with CitCRT $\left(2.45 \times 10^{-7}\right)$ was 10,000 -fold lower than that with native CRT $\left(2.05 \times 10^{-3}\right)$.

It is worth noting that, unlike native CRT, CitCRT transduced much more potent SE-activated signals when added to tissue cultures at lower concentrations (Figure 1C). The reason for this "paradoxical" dose-

Table 1. Kinetics of CRT-ligand interactions*

\begin{tabular}{lll}
\hline & \multicolumn{1}{c}{ Native CRT } & Cit-CRT \\
\hline $65-79^{*} 0401$ & & \\
$K_{\mathrm{a}}(1 / M \cdot$ second $)$ & $8.7 \pm 6.2$ & $560 \pm 0.4$ \\
$K_{\mathrm{d}}(1 / \mathrm{second})$ & $8.2 \pm 2.1 \times 10^{-4}$ & $5.1 \pm 0.2 \times 10^{-5}$ \\
$K_{\mathrm{D}}(M)$ & $9.6 \pm 3.8 \times 10^{-6}$ & $8.4 \pm 0.3 \times 10^{-8}$ \\
$\mathrm{C} 1 \mathrm{q}$ & & \\
$K_{\mathrm{a}}(1 / M \cdot$ second $)$ & $1.7 \pm 2.0 \times 10^{5}$ & $2.7 \pm 0.5 \times 10^{5}$ \\
$K_{\mathrm{d}}(1 /$ second $)$ & $2.8 \pm 0.2 \times 10^{-4}$ & $2.4 \pm 0.4 \times 10^{-4}$ \\
$K_{\mathrm{D}}(M)$ & $1.7 \pm 0.2 \times 10^{-9}$ & $9.0 \pm 1.1 \times 10^{-10}$ \\
\hline
\end{tabular}

*Values are the mean \pm SEM. CRT $=$ calreticulin.

response curve is unclear. One possible explanation is that at high tissue culture concentrations, there is saturation of the CRT-anchoring sites at the cell surface, with resultant higher abundance of free Cit-CRT in the supernatant. Due to its higher affinity, unbound CitCRT could conceivably "intercept" the SE ligand before it binds to the cell surface-anchored signal-transducing receptor.

The impact of individual arginine residues. Given that citrullination involves conversion of positively charged arginine residues to neutrally charged citrullines, we sought to determine whether the augmented interaction between the SE and Cit-CRT described above can be attributed to a loss of positive charge near the SE binding site, previously mapped to the CRT P-domain (8). Of the 8 arginine residues in the mature CRT protein, only $\mathrm{Arg}^{205}$ and $\mathrm{Arg}^{261}$ are located in its P-domain. Another remote, but possibly spatially relevant, residue is $\operatorname{Arg}^{160}$, located in the CRT $\mathrm{N}$-domain, close to its junction with the P-domain. MS

Table 2. MS analysis of CRT citrullination sites*

\begin{tabular}{|c|c|}
\hline Origin & Citrullinated sites \\
\hline \multicolumn{2}{|l|}{ Recombinant protein $\dagger$} \\
\hline Mouse Crt & $\operatorname{Arg}^{19}, \operatorname{Arg}^{160}, \operatorname{Arg}^{205}, \operatorname{Arg}^{261}$ \\
\hline \multicolumn{2}{|l|}{ FLS $\ddagger$} \\
\hline RA1 & $\operatorname{Arg}^{19}, \operatorname{Arg}^{56}, \operatorname{Arg}^{145}$ \\
\hline RA2 & $\operatorname{Arg}^{56}, \operatorname{Arg}^{160}, \operatorname{Arg}^{205}$ \\
\hline OA1 & $\mathrm{Arg}^{56}, \mathrm{Arg}^{205}$ \\
\hline $\mathrm{OA} 2$ & $\mathrm{Arg}^{56}, \mathrm{Arg}^{205}$ \\
\hline \multicolumn{2}{|l|}{ Synovial tissue $\ddagger$} \\
\hline RA3 & $\operatorname{Arg}^{56}, \operatorname{Arg}^{145}, \operatorname{Arg}^{205}$ \\
\hline RA4 & $\operatorname{Arg}^{19}, \operatorname{Arg}^{56}, \operatorname{Arg}^{145}, \operatorname{Arg}^{205}$ \\
\hline RA5 & $\operatorname{Arg}^{19}, \operatorname{Arg}^{205}, \operatorname{Arg}^{261}$ \\
\hline OA3 & $\operatorname{Arg}^{56}, \operatorname{Arg}^{145}, \operatorname{Arg}^{160}, \operatorname{Arg}^{205}$ \\
\hline OA4 & $\operatorname{Arg}^{19}, \operatorname{Arg}^{56}, \operatorname{Arg}^{145}, \operatorname{Arg}^{160}, \operatorname{Arg}^{205}, \operatorname{Arg}^{261}$ \\
\hline
\end{tabular}

$*$ FLS $=$ fibroblast-like synoviocyte; RA1 = rheumatoid arthritis patient 1 ; OA1 = osteoarthritis patient 1 .

$\dagger$ Recombinant mouse calreticulin (CRT) was citrullinated in vitro. CRT citrullination sites were identified by matrix-assisted laser desorption ionization-time-of-flight/time-of-flight mass spectrometry (MS). $\ddagger$ CRT citrullination sites in cell or tissue extracts were identified by liquid chromatography electrospray ionization MS/MS. 
analysis of the Cit-CRT used in Figure 1 showed that these 3 arginine residues were all citrullinated (Table 2). We therefore theorized that one or more of them may have contributed to the effects of Cit-CRT. Since citrulline is not a genetically coded residue, selective insertion of individual citrulline residues by recombinant DNA technology is not possible. We therefore adapted a site-directed citrulline-simulation approach, by mutating positively charged arginine residues to neutrally charged alanines. These substitutions did not introduce conformational changes in CRT, as evidenced by circular dichroism (see Supplementary Figure 1, available on the Arthritis \& Rheumatism web site at http://onlinelibrary. wiley.com/doi/10.1002/art.37814/abstract).

Wild-type CRT or its point mutant variants were immobilized on biosensor chips as described above, and their binding to the SE peptide ligand or HLA-DR tetrameric proteins was determined. As Figure 2 shows, a single arginine-to-alanine substitution in position 205 (R205A) augmented CRT binding to SE-expressing peptides (Figure 2A) and tetrameric proteins (Figure 2B), while R261A demonstrated diminished binding. R160A mutation did not have a significant impact on CRT-SE binding. None of the substitutions had a significant impact on CRT interaction with SE-negative peptides or tetramers, confirming the specificity of the findings. Interestingly, the $2 \mathrm{P}$-domain arginines, $\mathrm{Arg}^{205}$ and $\mathrm{Arg}^{261}$, had a diametrically opposite effect on SE binding. To further characterize the relative role of these arginines, we generated a double mutant expressing both substitutions (R205A/R261A). As can be seen in Figure 2 , the double mutant R205A/R261A retained an enhanced interaction with the SE, indicating that $\mathrm{Arg}^{205}$ had a dominant effect.

We next determined the relative role of individual P-domain arginines on SE-activated signaling. As can be seen in Figure 2D, the R205A point substitution significantly enhanced SE-activated signaling, while other mutations had no significant effect on SE signaling. Collectively, these findings identify the positive charge of $\operatorname{Arg}^{205}$ as a critical determinant of CRT function. Neutralization of this single charge led to markedly enhanced receptor-ligand interaction and signaling. Thus, deimination of $\operatorname{Arg}^{205}$ is the single most likely protein modification responsible for the effect of global CRT citrullination. Deimination of the 2 other arginines, $\operatorname{Arg}^{160}$ and $\mathrm{Arg}^{261}$, does not appear to contribute meaningfully to the net effect of global CRT citrullination on its SE receptor function.

Overabundance of Cit-CRT in RA synovium. As a prelude to future investigation of the clinical relevance of the data discussed above, we sought to determine the
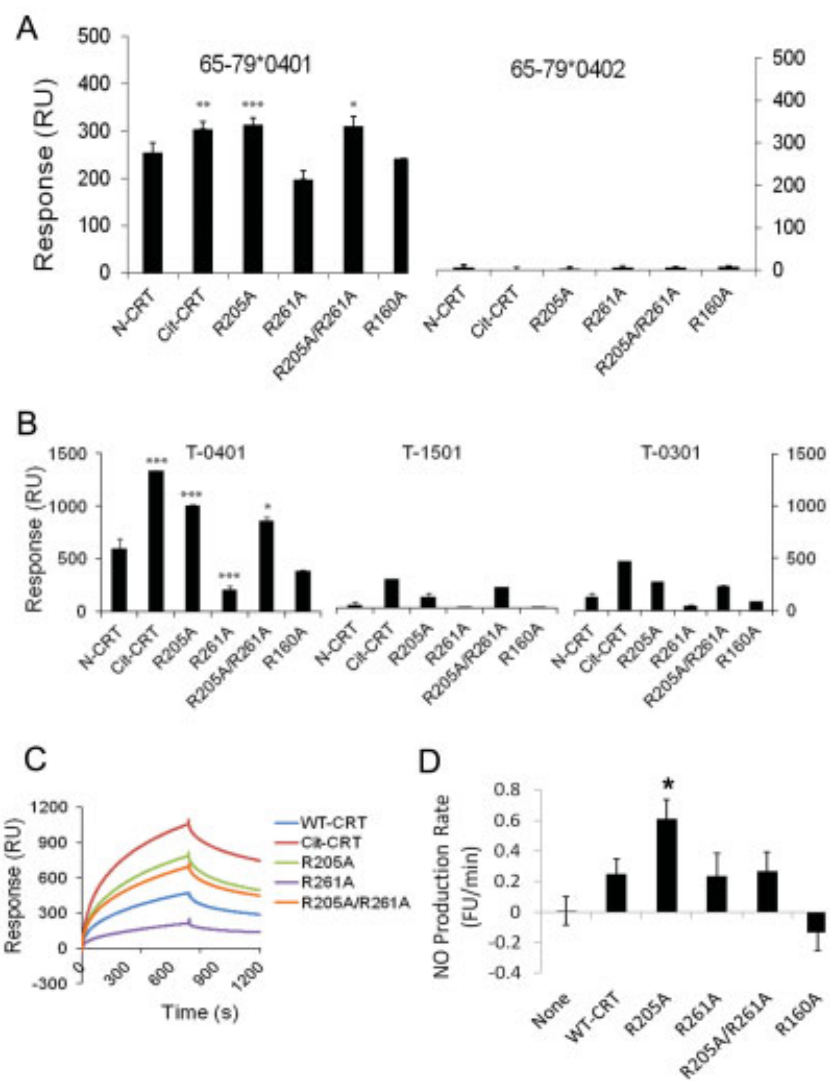

Figure 2. Role of individual CRT arginine residues. A, SPR data showing binding interactions between native CRT, Cit-CRT, or CRT with selected Arg-Ala substitutions and the SE-positive peptide ligand $65-79 * 0401$ or the control SE-negative peptide $65-79 * 0402$. The CRT variants were immobilized on a biosensor chip at 3,000 RU, 65$79 * 0401$ and $65-79 * 0402$ were each applied in the analyte at $330 \mu M$, and binding interactions were determined. B, SPR data showing binding interactions between the different CRT variants shown in A and SE-positive (T-0401) or SE-negative (T-1501 and T-0301) tetramers each applied in the analyte at a $250 \mathrm{n} M$ concentration. C, Representative sensorgrams of the data shown in B for binding with T-0401. D, NO production by CRT-deficient cells incubated with CRT or its variants and the SE-positive peptide $65-79 * 0401$. Data are representative of at least 2 independent identical experiments. Values in A, B, and $\mathbf{D}$ are the mean \pm SEM. $*=P<0.05 ; * *=P<0.01 ; * * *=P<$ 0.005 , versus native CRT in $\mathbf{A}$ and $\mathbf{B}$ and versus wild-type CRT (WT-CRT) in D. See Figure 1 for other definitions.

abundance of Cit-CRT in vivo. Figure 3A shows 2-D protein gels of FLS extracts obtained from representative RA and OA patients and blotted with anticitrulline antibodies. A citrullinated acidic protein spot (pI 4.3) fitting the expected CRT coordinates was found to be much more abundant in the RA sample than in the OA sample. When membranes were stripped and blotted with anti-CRT, the identity of that spot was confirmed as CRT. The spot in the RA sample showed a unique acidic 

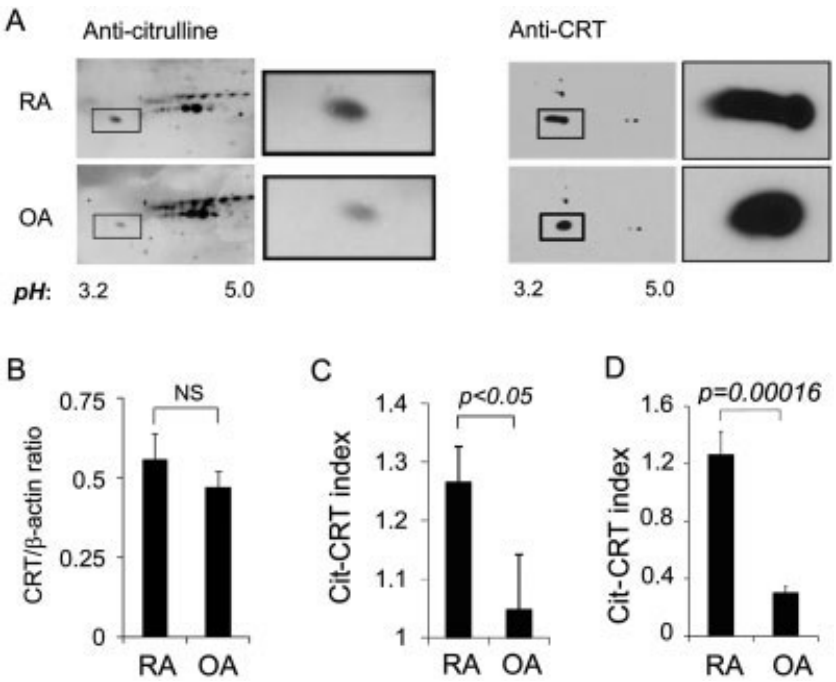

Figure 3. Quantification of synovial Cit-CRT. A, Representative 2-dimensional (2-D) immunoblot display of protein citrullination patterns in fibroblast-like synoviocytes (FLS) obtained from patients with rheumatoid arthritis (RA) or osteoarthritis (OA). Left, An acidic protein fitting CRT 2-D coordinates (boxed area) was found to be overexpressed in RA. Enlarged images of the spots are shown. Right, Membranes were stripped and reprobed with anti-CRT antibodies. The spots (boxed areas) were confirmed as CRT. Enlarged images of the CRT spots show an acidic shift in the RA sample, consistent with citrullination. B, CRT: $\beta$-actin ratio in synovial tissue samples obtained from patients with RA and patients with OA during joint replacement surgery. Total protein extracts were separated by polyacrylamide gel electrophoresis and blotted with anti-CRT and anti- $\beta$-actin. Levels of total CRT relative to $\beta$-actin in crude synovial tissue extracts were not statistically different between RA and OA samples. C, Cit-CRT index (the ratio between Cit-CRT and total CRT) in synovial tissue samples obtained from patients with RA and patients with OA. Abundance of Cit-CRT in synovial tissue was significantly higher in RA than in OA samples. D, Cit-CRT index in FLS samples obtained from patients with RA and patients with OA. Quantification of Cit-CRT in FLS was performed as described in part C. Values in $\mathbf{B}-\mathbf{D}$ are the mean \pm SEM ( $\mathrm{n}=4$ patients with RA and 5 patients with OA). See Figure 1 for other definitions.

shift, a feature which is characteristic of citrullinated proteins.

There was no statistically significant difference in total CRT abundance between 5 RA and 4 OA synovial tissue samples (Figure 3B). However, as shown in Figure 3C, RA synovial tissue had a significantly higher relative abundance of Cit-CRT compared with OA tissue. We performed a similar analysis on 22 FLS cell lines. As shown in Figure 3D, RA FLS had a significantly higher abundance of Cit-CRT compared to OA FLS.

We have also used MS analysis to determine citrullination changes. Deimination of 1 arginine to citrulline results in a mass shift of 1 dalton, which is difficult to detect by standard MS. To overcome this limitation, we used a chemical modification method, which involves a reaction of the ureido group of citrulline with 2,3-butanedione in the presence of antipyrine (29). This results in a characteristic mass shift of +238 daltons, which is easier to detect. MS analyses were performed on samples from 5 RA and 4 OA patients (Table 2). There was some variability among individual samples in the repertoire of citrullination sites; however, we could not identify qualitative differences between RA and OA samples in this early study. It remains possible that while there are no qualitative differences between RA and OA in terms of the repertoire of citrullinated sites, there could be a quantitative difference, namely differential abundance of site-specific deimination. Demonstration of such differences is likely to require analysis of a very large number of samples.

\section{DISCUSSION}

The basis of the association between RA and the $\mathrm{SE}$ and the role of protein citrullination in the disease are both unknown. We have previously demonstrated that the SE is a ligand that interacts with cell surface $\mathrm{CRT}$ at a specific binding site in the receptor P-domain and transduces innate immune signals. In this study we demonstrated that citrullination of CRT increased its affinity for the SE ligand and enhanced its signaling potency. By introducing citrullination-mimicking sitespecific mutations, we were able to demonstrate that particular arginine residues in the vicinity of the SE binding site play distinct modulatory roles in SE-CRT interaction and signal transduction. Further, we demonstrated, for the first time, that Cit-CRT is overabundant in RA synovial tissue and synovial tissue-derived FLS.

The findings of this study have dual significance. First, they provide important insights into the molecular topology and tridimensional confines of SE-CRT interaction, and second, they offer a plausible model that could explain the pathogenic role of the SE in RA and the interplay between genetic and nongenetic factors in the disease.

The impact of citrullination on the SE-CRT interaction observed here is consistent with previous reports concerning structural and functional effects of this posttranslational protein modification. Citrullination involves deimination of arginine with resultant conversion to citrulline. Arginine is strongly basic, whereas citrulline is a charge-neutral residue. Citrullination therefore introduces net loss in the protein charge, and consequently could affect interaction with proteins and other biomolecules (30-33). In addition to the effect of deimination on the polypeptide primary sequence, 
citrullinated proteins can undergo secondary conformational changes that influence their access to other molecules (34).

Of the two mechanisms by which protein citrullination can affect interaction with other molecules, we tend to favor loss of charge as an underlying mechanism. As circular dichroism spectra indicate, CRT citrullination did not produce a significant conformational change (see Supplementary Figure 1, available on the Arthritis \& Rheumatism web site at http://onlinelibrary.wiley.com/ doi/10.1002/art.37814/abstract). Further, the R205A mutant, with a single substitution, reproduced the effect of global citrullination, suggesting that a charged moiety near the SE binding site accounts for most of the citrullination effect on CRT-SE interaction. Based on the finding that single substitutions at $\operatorname{Arg}^{160}$ and $\operatorname{Arg}^{261}$ had a neutral or an opposite effect, respectively, we propose that citrullinated $\mathrm{Arg}^{205}$ may have a dominant SE binding enhancing effect, which overrides an opposite effect by the more remote $\mathrm{Arg}^{261}$. A proposed model of the spatial role of P-domain arginine residues on SE binding is depicted in Figure 4.

Consistent with the SE ligand hypothesis (35-37), the affinity of SE-CRT interaction correlated with signaling potency. Cit-CRT, which displayed higher affinity for the SE ligand in a cell-free binding assay, transduced a markedly more potent signal for production of $\mathrm{NO}$ than did native CRT. Likewise, CRT mutant R205A, which demonstrated higher SE binding, transduced more potent SE signaling, while R160A, which did not significantly affect the interaction with the SE, also did not produce a significant signaling effect. An interesting exception to this rule was seen with CRT mutant R261A, which, despite its significantly weaker interaction with the SE ligand in cell-free binding assays, maintained signal transduction potency comparable to that of wildtype CRT. The reason is unclear, but could conceivably be related to the fact that the $\operatorname{Arg}^{261}$ side chain is directed away from the SE binding site (Figure 4). This dichotomy suggests that citrullination of $\operatorname{Arg}^{261}$ is less likely to impact SE biologic effects in physiologic conditions. The specificity of the impact of citrullination changes around the SE binding site indicate that augmented SE-CRT binding, rather than nonspecific increased signaling by Cit-CRT, is responsible for the observed effect on SE-activated signaling.

We found no measurable differences in the abundance of total CRT between OA and RA samples. However, the fraction of Cit-CRT of total CRT was significantly higher in RA. These data corroborate and expand on a brief prior observation that Cit-CRT is overexpressed in the serum of patients with early RA

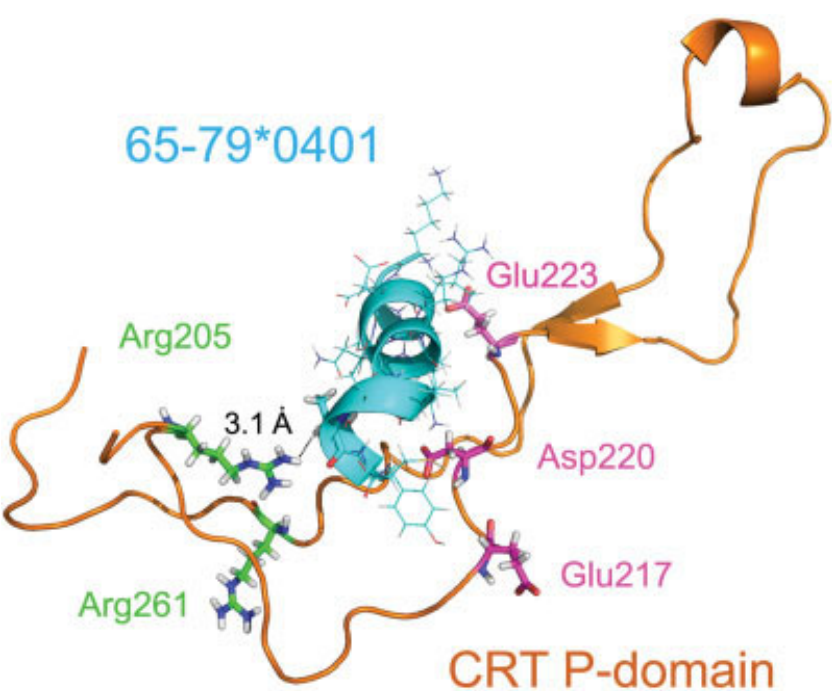

Figure 4. A model of CRT-SE interaction. The SE ligand (cyan) interacts with $\mathrm{Glu}^{217}, \mathrm{Asp}^{220}$ and $\mathrm{Glu}^{223}$ (pink), previously identified as the SE binding site in the CRT P-domain (orange) (8). P-domain $\mathrm{Arg}^{205}$ and $\mathrm{Arg}^{261}$ are shown in green. The CRT $\operatorname{Arg}^{205}$ side chain points toward the SE, and reaches a distance of $3.1 \AA$ from the SE. The model proposed here predicts that this positively charged moiety exerts an inhibitory effect on CRT-SE interaction. Citrullination of $\mathrm{Arg}^{205}$ may therefore decrease a putative hindrance and increase binding affinity. $\mathrm{Arg}^{261}$, in contrast, points away from the SE binding site at a predicted distance of $12.9 \AA$, in an orientation that cannot inhibit SE-CRT interaction. We propose that the enhancing effect of $\mathrm{Arg}^{261}$ on SE-CRT binding could be related to charge-based interaction with other parts of the HLA-DR molecule, or a stabilizing effect on CRT spatial conformation. The functionally inert residue $\mathrm{Arg}^{160}$ is located in the N-domain at a distance of $24.7 \AA$ from the SE binding site (not shown). See Figure 1 for definitions.

(38). The data shown here are the first indication that the synovial tissue itself, the primary disease focus in RA, overexpresses this posttranslationally modified protein. It is important to note that abundance of citrullinated proteins in pathologic tissue is not unique to RA, and has been reported in many other conditions, such as multiple sclerosis, Alzheimer's disease, and Crohn's disease $(11,39)$. The cause of excessive protein citrullination in pathologic tissue is unclear, but could be a consequence of cell death (40) or oxidative stress (41).

Citrullinated proteins may contribute directly to the disease process, rather than be only a consequence of inflammation (42). However, unlike all other conditions in which citrullinated proteins have been found, in RA these proteins act as neoantigens and trigger an autoantibody response (ACPA). The strong association between ACPAs and RA, the appearance of these autoantibodies prior to disease onset, and their association with SE and smoking have led many to suggest that ACPAs may play a direct pathogenic role in RA (43). 
Some evidence in support of this hypothesis has been documented in experimental murine models of arthritis $(44,45)$.

It has previously been reported that SEexpressing HLA-DR molecules can bind and present citrullinated peptides $(46,47)$. Given that observation, could Cit-CRT peptides be preferentially presented by SE-expressing DR molecules in RA and generate ACPAs? The findings of this study cannot refute or substantiate that scenario. However, it is important to point out that presentation of Cit-CRT peptides is not the underlying mechanism of our findings for several reasons. First, we used different allele-specific tetramers, all of which contain a uniform, covalently bound groove CLIP peptide. It is highly unlikely that these tetramers would be able to present other peptides. Likewise, synthetic SE peptides in solution are incapable of antigen presentation. Second, we are not aware of reports of autoantibodies to Cit-CRT, while there are many other Cit-peptides that are known to be immunogenic in RA (citrullinated fibrinogen, citrullinated vimentin, etc.). We therefore believe it is not necessary to postulate that Cit-CRT might be the autoantigen that drives the anti-cyclic citrullinated peptide response.

This study did not set out to explain the genesis of ACPAs, and therefore we cannot determine the causeeffect relationship between SE-activated signaling and ACPAs. However, it should be pointed out that in a recent study we demonstrated that the SE-CRT pathway leads to immune dysregulation that diminishes Treg cell numbers, and reciprocally enhances Th17 expansion (7). It remains to be determined whether SE-mediated immune dysregulatory effect can facilitate autoimmune responses, such as ACPAs.

Independent of the putative pathogenic role of ACPAs, the findings of this study could help to better explain the interaction between inherited and acquired factors in RA. Epidemiologic data strongly suggest that an interaction between genetic (SE) and nongenetic factors may be involved. Here we identified an acquired, possibly environmentally induced event that substantially amplified the functional effect of a genetically determined factor, the SE. It is therefore tempting to propose that the SE functional effect in RA may be facilitated by site-specific citrullinated CRT. Thus, in healthy individuals carrying SE-encoding HLA-DRB1 alleles, the SE ligand interacts at low affinity with cell surface CRT that is largely noncitrullinated. This interaction could be beneficial by activating low-grade Th17 polarization (7), which could be advantageous against pathogens (48). As a result of environmental stresses (for example, long-term exposure to cigarette smoke), there could be a gradual activation of PAD with resultant citrullination of many proteins, including CRT. As the abundance of Cit-CRT gradually increases, it could reach a critical threshold, when the affinity to the SE results in exaggerated signaling with resultant pathology. In individuals with the right constellation of genetic risk factors, the combined scenario described above could trigger disease onset.

\section{ACKNOWLEDGMENTS}

We thank Dr. Steven Lundy for helpful discussions and Ms Gail Quaderer for administrative support.

\section{AUTHOR CONTRIBUTIONS}

All authors were involved in drafting the article or revising it critically for important intellectual content, and all authors approved the final version to be published. Dr. Holoshitz had full access to all of the data in the study and takes responsibility for the integrity of the data and the accuracy of the data analysis.

Study conception and design. Ling, Fox, Holoshitz.

Acquisition of data. Ling, Cline, Haug.

Analysis and interpretation of data. Ling, Cline, Haug, Fox, Holoshitz.

\section{REFERENCES}

1. Gregersen PK, Silver J, Winchester RJ. The shared epitope hypothesis: an approach to understanding the molecular genetics of susceptibility to rheumatoid arthritis. Arthritis Rheum 1987;30: 1205-13.

2. Turesson C, Schaid DJ, Weyand CM, Jacobsson LT, Goronzy JJ, Petersson, IF, et al. The impact of HLA-DRB1 genes on extraarticular disease manifestations in rheumatoid arthritis. Arthritis Res Ther 2005;7:R1386-93.

3. Moreno I, Valenzuela A, Garcia A, Yelamos J, Sanchez, B, Hernanz W. Association of the shared epitope with radiological severity of rheumatoid arthritis. J Rheumatol 1996;23:6-9.

4. Ling S, Lai A, Borschukova O, Pumpens P, Holoshitz J. Activation of nitric oxide signaling by the rheumatoid arthritis shared epitope. Arthritis Rheum 2006;54:3423-32.

5. Ling S, Li Z, Borschukova O, Xiao L, Pumpens P, Holoshitz J. The rheumatoid arthritis shared epitope increases cellular susceptibility to oxidative stress by antagonizing an adenosine-mediated anti-oxidative pathway. Arthritis Res Ther 2007;9:R5.

6. Ling S, Pi X, Holoshitz J. The rheumatoid arthritis shared epitope triggers innate immune signaling via cell surface calreticulin. J Immunol 2007;179:6359-67.

7. De Almeida DE, Ling S, Pi X, Hartmann-Scruggs AM, Pumpens $\mathrm{P}$, Holoshitz J. Immune dysregulation by the rheumatoid arthritis shared epitope. J Immunol 2010;185:1927-34.

8. Ling S, Cheng A, Pumpens P, Michalak M, Holoshitz J. Identification of the rheumatoid arthritis shared epitope binding site on calreticulin. PLoS One 2010;5:e11703.

9. Silman AJ, MacGregor A, Thomson W, Holligan S, Carthy D, Ollier WE. Twin concordance rates for rheumatoid arthritis: results of a nationwide study. Br J Rheumatol 1993;32:903-7.

10. Jungel A, Ospelt C, Gay S. What can we learn from epigenetics in the year 2009? Curr Opin Rheumatol 2010;22:284-92.

11. Makrygiannakis D, af Klint E, Lundberg IE, Lofberg R, Ulfgren AK, Klareskog L, et al. Citrullination is an inflammationdependent process. Ann Rheum Dis 2006;65:1219-22.

12. Vossenaar ER, Smeets TJ, Kraan MC, Raats JM, van Venrooij 
WJ, Tak PP. The presence of citrullinated proteins is not specific for rheumatoid synovial tissue. Arthritis Rheum 2004;50:3485-94.

13. Masson-Bessiere C, Sebbag M, Girbal-Neuhauser E, Nogueira L, Vincent C, Senshu T, et al. The major synovial targets of the rheumatoid arthritis-specific antifilaggrin autoantibodies are deiminated forms of the $\alpha$ - and $\beta$-chains of fibrin. J Immunol 2001;166:4177-84.

14. Matsuo K, Xiang Y, Nakamura H, Masuko K, Yudoh K, Noyori K, et al. Identification of novel citrullinated autoantigens of synovium in rheumatoid arthritis using a proteomic approach. Arthritis Res Ther 2006;8:R175.

15. Chang X, Yamada R, Suzuki A, Kochi Y, Sawada T, Yamamoto $\mathrm{K}$. Citrullination of fibronectin in rheumatoid arthritis synovial tissue. Rheumatology (Oxford) 2005;44:1374-82.

16. Klareskog L, Ronnelid J, Lundberg K, Padyukov L, Alfredsson L. Immunity to citrullinated proteins in rheumatoid arthritis. Annu Rev Immunol 2008;26:651-75.

17. Huizinga TW, Amos CI, van der Helm-van Mil AH, Chen W, van Gaalen FA, Jawaheer D, et al. Refining the complex rheumatoid arthritis phenotype based on specificity of the HLA-DRB1 shared epitope for antibodies to citrullinated proteins. Arthritis Rheum 2005;52:3433-8.

18. Padyukov L, Silva C, Stolt P, Alfredsson L, Klareskog L, for the Epidemiological Investigation of Rheumatoid Arthritis Study Group. A gene-environment interaction between smoking and shared epitope genes in HLA-DR provides a high risk of seropositive rheumatoid arthritis. Arthritis Rheum 2004;50:3085-92.

19. Pedersen M, Jacobsen S, Garred P, Madsen HO, Klarlund M, Svejgaard A, et al. Strong combined gene-environment effects in anti-cyclic citrullinated peptide-positive rheumatoid arthritis: a nationwide case-control study in Denmark. Arthritis Rheum 2007;56:1446-53.

20. Karlson EW, Chang SC, Cui J, Chibnik LB, Fraser PA, Devivo I, et al. Gene-environment interaction between HLA-DRB1 shared epitope and heavy cigarette smoking in predicting incident RA. Ann Rheum Dis 2010;69:54-60.

21. Makrygiannakis D, Hermansson M, Ulfgren AK, Nicholas AP, Zendman AJ, Eklund A, et al. Smoking increases peptidylarginine deiminase 2 enzyme expression in human lungs and increases citrullination in BAL cells. Ann Rheum Dis 2008;67:1488-92.

22. Tran CN, Davis MJ, Tesmer LA, Endres JL, Motyl CD, Smuda C, et al. Presentation of arthritogenic peptide to antigen-specific $\mathrm{T}$ cells by fibroblast-like synoviocytes. Arthritis Rheum 2007;56: 1497-506.

23. Arnett FC, Edworthy SM, Bloch DA, McShane DJ, Fries JF, Cooper NS, et al. The American Rheumatism Association 1987 revised criteria for the classification of rheumatoid arthritis. Arthritis Rheum 1988;31:315-24.

24. Mesaeli N, Nakamura K, Zvaritch E, Dickie P, Dziak E, Krause $\mathrm{KH}$, et al. Calreticulin is essential for cardiac development. J Cell Biol 1999;144:857-68.

25. Vossenaar ER, Nijenhuis S, Helsen MM, van der Heijden A, Senshu T, van den Berg WB, et al. Citrullination of synovial proteins in murine models of rheumatoid arthritis. Arthritis Rheum 2003;48:2489-500.

26. Holm A, Rise F, Sessler N, Sollid LM, Undheim K, Fleckenstein B. Specific modification of peptide-bound citrulline residues. Anal Biochem 2006;352:68-76.

27. Vivekanandan-Giri A, Slocum JL, Buller CL, Basrur V, Ju W, Pop-Busui R, et al. Urine glycoprotein profile reveals novel markers for chronic kidney disease. Int J Proteomics 2011;214715.

28. Goicoechea S, Pallero MA, Eggleton P, Michalak M, MurphyUllrich JE. The anti-adhesive activity of thrombospondin is mediated by the N-terminal domain of cell surface calreticulin. J Biol Chem 2002;277:37219-28.

29. Obeid M, Tesniere A, Ghiringhelli F, Fimia GM, Apetoh L,
Perfettini JL, et al. Calreticulin exposure dictates the immunogenicity of cancer cell death. Nat Med 2007;13:54-61.

30. Gyorgy B, Toth E, Tarcsa E, Falus A, Buzas EI. Citrullination: a posttranslational modification in health and disease. Int $\mathrm{J}$ Biochem Cell Biol 2006;38:1662-77.

31. Senshu T, Akiyama K, Nomura K. Identification of citrulline residues in the $\mathrm{V}$ subdomains of keratin $\mathrm{K} 1$ derived from the cornified layer of newborn mouse epidermis. Exp Dermatol 1999; 8:392-401.

32. Guo Q, Fast W. Citrullination of inhibitor of growth 4 (ING4) by peptidylarginine deminase 4 (PAD4) disrupts the interaction between ING4 and p53. J Biol Chem 2011;286:17069-78.

33. Inagaki M, Takahara $\mathrm{H}$, Nishi $Y$, Sugawara K, Sato C. $\mathrm{Ca}^{2+}$. dependent deimination-induced disassembly of intermediate filaments involves specific modification of the amino-terminal head domain. J Biol Chem 1989;264:18119-27.

34. Pritzker LB, Joshi S, Gowan JJ, Harauz G, Moscarello MA. Deimination of myelin basic protein. 1. Effect of deimination of arginyl residues of myelin basic protein on its structure and susceptibility to digestion by cathepsin D. Biochemistry 2000;39: $5374-81$.

35. Holoshitz J, De Almeida DE, Ling S. A role for calreticulin in the pathogenesis of rheumatoid arthritis. Ann N Y Acad Sci 2010; 1209:91-8.

36. De Almeida DE, Ling S, Holoshitz J. New insights into the functional role of the rheumatoid arthritis shared epitope. FEBS Lett 2011;585:3619-2.

37. De Almeida DE, Holoshitz J. MHC molecules in health and disease: at the cusp of a paradigm shift. Self Nonself 2011;2:43-8.

38. Goeb V, Thomas-L'Otellier M, Daveau R, Charlionet R, Fardellone $\mathrm{P}$, Le Loet $\mathrm{X}$, et al. Candidate autoantigens identified by mass spectrometry in early rheumatoid arthritis are chaperones and citrullinated glycolytic enzymes. Arthritis Res Ther 2009;11:R38.

39. Ishigami A, Ohsawa T, Hiratsuka M, Taguchi H, Kobayashi S, Saito Y, et al. Abnormal accumulation of citrullinated proteins catalyzed by peptidylarginine deiminase in hippocampal extracts from patients with Alzheimer's disease. J Neurosci Res 2005;80: $120-8$.

40. De Ceuleneer M, Van Steendam K, Dhaenens M, Deforce D. In vivo relevance of citrullinated proteins and the challenges in their detection. Proteomics 2012;12:752-60.

41. Neeli I, Kahn SN, Radic M. Histone deimination as a response to inflammatory stimuli in neutrophils. J Immunol 2008;180:1895-902.

42. Musse AA, Harauz G. Molecular "negativity" may underlie multiple sclerosis: role of the myelin basic protein family in the pathogenesis of MS. Int Rev Neurobiol 2007;79:149-72.

43. Uysal H, Nandakumar KS, Kessel C, Haag S, Carlsen S, Burkhardt $\mathrm{H}$, et al. Antibodies to citrullinated proteins: molecular interactions and arthritogenicity. Immunol Rev 2010;233:9-33.

44. Hill JA, Bell DA, Brintnell W, Yue D, Wehrli B, Jevnikar AM, et al. Arthritis induced by posttranslationally modified (citrullinated) fibrinogen in DR4-IE transgenic mice. J Exp Med 2008;205:967-79.

45. Harre U, Georgess D, Bang H, Bozec A, Axmann R, Ossipova E, et al. Induction of osteoclastogenesis and bone loss by human autoantibodies against citrullinated vimentin. J Clin Invest 2012; 122:1791-802.

46. James EA, Moustakas AK, Bui J, Papadopoulos GK, Bondinas G, Buckner JH, et al. HLA-DR1001 presents "altered-self" peptides derived from joint-associated proteins by accepting citrulline in three of its binding pockets. Arthritis Rheum 2010;62:2909-18.

47. Hill JA, Bell DA, Brintnell W, Yue D, Wehrli B, Jevnikar AM, et al. Arthritis induced by posttranslationally modified (citrullinated) fibrinogen in DR4-IE transgenic mice. J Exp Med 2008; 205:967-79.

48. Curtis MM, Way SS. Interleukin-17 in host defense against bacterial, mycobacterial and fungal pathogens. Immunology 2009; 126:177-85. 